\title{
A Construction of Imprimitive Groups of Rank 4 or 5
}

\author{
Chang Wang*, Renbing Xiao \\ School of Mathematics, Yunnan Normal University, Kunming, China \\ Email address: \\ 1491822991@qq.com (Chang Wang), 1341053221@qq.com (Renbing Xiao) \\ ${ }^{*}$ Corresponding author
}

\section{To cite this article:}

Chang Wang, Renbing Xiao. A Construction of Imprimitive Groups of Rank 4 or 5. Applied and Computational Mathematics. Vol. 9, No. 6, 2020, pp. 175-178. doi: 10.11648/j.acm.20200906.11

Received: September 15, 2020; Accepted: October 23, 2020; Published: November 4, 2020

\begin{abstract}
Let $\mathrm{G}$ be a transitive permutation group acting on a finite set $\Omega$. For a point $\alpha$ of $\Omega$, the set of the images of $\mathrm{G}$ acting on $\alpha$ is called the orbit of $\alpha$ under $G$ and is denoted by $\alpha^{\mathrm{G}}$, and the set of elements in $\mathrm{G}$ which fix $\alpha$ is called the stabilizer of $\alpha$ in $\mathrm{G}$ and is denoted by $\mathrm{G}_{\alpha}$. We can get some new orbits by using the natural action of the stabilizer $\mathrm{G}_{\alpha}$ on $\Omega$, and then we can define the suborbit of $\mathrm{G}$. The suborbits of $\mathrm{G}$ on $\Omega$ are defined as the orbits of a point stabilizer on $\Omega$. The number of suborbits is called the rank of $G$ and the length of suborbits is called the subdegree of $G$. For finite primitive groups, the study of the rank and subdegrees of group has a long history. In this paper, we construct a class of imprimitive permutation groups of rank 4 or 5 by using imprimitive action and product action of wreath product, determine the number and the length of the suborbits, and extend the results to imprimitive permutation groups of rank $m+1$ and $2^{\mathrm{n}}+1$, where $m$ and $n$ are positive integers.
\end{abstract}

Keywords: Permutation Group, Transitive Action, Rank, Suborbit

\section{Introduction}

Let $\mathrm{G}$ be a transitive permutation group acting on a finite set $\Omega$. The suborbits of $\mathrm{G}$ on $\Omega$ are defined to be the orbits of the stabilizer $\mathrm{G}_{\alpha}$ acting on $\Omega$, where $\alpha \in \Omega$. The number of the suborbits is called the rank of $G$, and the lengths of the suborbits are called the subdegrees of G. For finite primitive groups, the study of the rank and subdegrees of such groups has a fairly long history, and the research mainly focuses on the case of small ranks and subdegrees. For this topic, many scholars have obtained some good results. It is clear that the rank of a transitive permutation group $G$ is 2 if and only if $G$ is 2-transitive. With the help of the finite simple group classification theorem, all finite 2-transitive permutation groups have been completely determined. Therefore, the study of the ranks of finite primitive groups is mainly focused the on case of rank $\geq 3$.

For a finite transitive group $\mathrm{G}$ of rank 3, Higman's review article [4] is a good resource for early work. The research on this problem is often combined with the research of the suborbits of the transitive permutation groups. When the length of an suborbit is large, this kind of problem is very difficult to study. Therefore the work so far has been focused mainly on the cases of suborbit $\leq 5$.

If a primitive permutation group has non-trivial suborbit of length 1, it must be a regular transitive group of prime degree. If a primitive permutation group has a suborbit of length 2 , it must be a dihedral group of prime degree, see [8, Theorem 5] for details. For a primitive permutation group has a suborbit of length 3 , the structure of the groups becomes very complex. Using the work of Sim [11], Wong [16] has completed the classification of these groups. For primitive permutation groups have a suborbit of length 4, the structure of the groups becomes more complex. Sim [11] and Quirin [10] obtained part of the result regarding the case. Finally J. Wang used the classification theorem of finite simple group to obtain a complete classification for these groups in literature [13]. In [6], Li, Liu and Marusic presented a new charaterization of primitive groups with suborbits of length 3 and 4, and the result is used to give a complete description of symmetric graphs of valency 3 or 4 . For primitive permutation groups containg a suborbit of length 5, J. Wang has obtained partial results in $[14,15]$. Recently in 2018, the classification of corresponding primitive groups was completed by Fawcett, Giudic, Li, Praeger, et, al in literature [3].

In recent years, much more attention has also been paid to the study of the ranks and subdegrees of imprimitive permutation groups. In this paper, we construct a class of imprimitive permutation groups with rank 4 or 5 , determine the length of the subdegrees, and extend the results to 
imprimitive permutation groups with rank $\mathrm{m}+1$ and $2^{\mathrm{n}}+1$, where $m$ and $n$ are positive integers.

\section{Preliminaries}

In this section we present some of the basic concepts and results of permutation groups which will be used in this paer. For more general information about permutation groups, see Dixon's classical monograph [2]. The following notation and terminology are come from [2].

Definition 2.1 Let $\mathrm{G}$ be a group and $\Omega$ be a nonempty set, and suppose that for each $\alpha \in \Omega$ and each $x \in G$ we have defined an element of $\Omega$ denoted by $\alpha^{\mathrm{x}}$. Then we say that this defines an action of $\mathrm{G}$ on $\Omega$ (or G acting on $\Omega$ ) if:

(1) $\alpha^{1}=\alpha$ for all $\alpha \in \Omega$ (Where 1 denotes the identity element of G; and,

(2) $\left(\alpha^{\mathrm{x}}\right)^{\mathrm{y}}=\alpha^{\mathrm{xy}}$ for all $\alpha \in \Omega$ and $\mathrm{x}, y \in G$.

Definition 2.2 When a group $\mathrm{G}$ acts on a set $\Omega$, a typical point $\alpha$ is moved by elements of $\mathrm{G}$ to various other points. The set of the images of $\mathrm{G}$ acting on $\mathrm{C}$ is called the orbit of $\alpha$ under $\mathrm{G}$, and we denote it by

$$
\alpha^{\mathrm{G}}:=\left\{\alpha^{\mathrm{x}} \mid \mathrm{x} \in \mathrm{G}\right\} .
$$

Definition 2.3 A group $G$ acting on a set $\Omega$ is said to be transitive on $\Omega$ If it has only one orbit, and so $\alpha^{\mathrm{G}}=\Omega$ for all $\alpha \in \Omega$.

Definition 2.4 Let $\mathrm{G}$ be a group acting transitively on a set $\Omega$, A nonempty subset $\Delta$ of $\Omega$ is called a block for $\mathrm{G}$ if for each $x \in G$ either $\Delta^{x}=\Delta$ or $\Delta^{x} \cap \Delta=\varnothing$.

A group acting transitively on $\Omega$ has $\Omega$ and the singletons $\{\alpha\}(\alpha \in \Omega)$ as blocks; these blocks are called the trivial blocks. Any other blocks (if any) are called nontrivial.

Definition 2.5 Let $\mathrm{G}$ be a group which acts transitively on $\Omega$. We say that the group is primitive if $\mathrm{G}$ has no nontrivial block on $\Omega$; Otherwise $\mathrm{G}$ is called imprimitive.

Definition 2.6 Let $\mathrm{G}$ be a group acting transitively on a set $\Omega$. Then $\mathrm{G}$ induces a natural action on the Cartesian product $\Omega \times \Omega=\{(\alpha, \beta) \mid \alpha, \beta \in \Omega\}$. The orbits of $G$ on this set are called the orbitals of $\mathrm{G}$ on $\Omega$.

There is a close relationship between the orbitals of $\mathrm{G}$ and the orbits of the point stabilizers of G. For each orbital $\Delta$ of G and each $\alpha \in \Omega$, we define

$$
\Delta(\alpha) \stackrel{\Delta}{=}\{\beta \mid \beta \in \Omega,(\alpha, \beta) \in \Delta\}
$$

It is easy to verify that the mapping $\Delta \mapsto \Delta(\alpha)$ is a bijection from the set of orbitals of $\mathrm{G}$ onto the set of orbits of $\mathrm{G}_{\alpha}$. In particular, the number of orbitals is equel to the number of orbits of $\mathrm{G}$; this number is called the rank of $\mathrm{G}$. An orbit of $\mathrm{G}_{\alpha}$ for any $\alpha \in \Omega$ is called a suborbit of $\mathrm{G}$.

\section{Imprimitive Permutation Group of Rank 4 or 5}

For research the permutation groups in this paper, we need to introduce the concepts of wreath product and semiproduct of groups. The basic concepts are fiven as follows.

The notion of a semidirect product of two groups generalizes the idea of a direct product.

Definition 3.1 Let $\mathrm{H}$ and $\mathrm{K}$ which respects the group structure on $\mathrm{K}$; so for each $x \in H$ the mapping $\mathrm{u} \mapsto u^{x}$ is an automorphism of K. Put

$$
G \stackrel{\Delta}{=}\{(u, x) \mid u \in K, x \in H\}
$$

and define a product on $\mathrm{G}$ by

$$
(u, x)(v, y)=\left(u v^{x^{-1}}, x y\right), \text { for all }(\mathrm{u}, \mathrm{x}),(\mathrm{v}, \mathrm{y}) \in \mathrm{G}
$$

Definition 3.2 Let $\Gamma=\{1,2, \cdots, \mathrm{m}\}$ and $\Delta$ be a nonempty, and $\operatorname{Fun}(\Gamma, \Delta)$ means a set of all mapping of $\Gamma$ to $\Delta$, then it can denoted by $\Delta^{\mathrm{m}}$. If $\Delta=\mathrm{K}$ is a group, the $\operatorname{Fun}(\Gamma, \mathrm{K})=\mathrm{K}^{\mathrm{m}}$ means the direct product of $\mathrm{K}$, Let $\mathrm{H} \leq \mathrm{S}_{\mathrm{m}}$, then $\mathrm{H}$ have a naturally action on $\Gamma=\{1,2, \ldots, \mathrm{m}\}$, so we can define an action of $\mathrm{H}$ on $\operatorname{Fun}(\Gamma, \mathrm{K})$ as follow:

For each $\left(k_{1}, k_{2}, \cdots, k_{m}\right) \in \mathrm{K}^{m}$, Let $x \in H$ and define:

$$
\left(k_{1}, k_{2}, \cdots, k_{m}\right)^{x}=\left(k_{1^{y}}, k_{2^{y}}, \cdots, k_{m^{y}}\right), \text { where } y=x^{-1},
$$

Then we can obtain a semidirect product $\operatorname{Fun}(\Gamma, \mathrm{K}): \mathrm{H}=$ $\mathrm{K}^{\mathrm{m}}$ : $\mathrm{H}$, which is called wreath product of $\mathrm{K}$ and $\mathrm{H}$ and denoted by $K w r_{\Gamma} H$.

Our first result is as follows.

Theorem 3.3 The group $\mathrm{G}=\mathrm{S}_{\mathrm{n}}$ wr $\mathrm{S}_{3}$ and its subgroups $\mathrm{S}_{\mathrm{n}}$ wr $C_{3}$, $\left(S_{n}\right.$ wr $\left.S_{3}\right) \cap A_{n}$, and $\left(S_{n}\right.$ wr $\left.C_{3}\right) \cap A_{n}$ all have an imprimitive action of degree $3 \mathrm{n}$, with rank 4 .

Proof Let $\Omega=\{1,2, \ldots, 3 n\}, \Delta_{1}=\{1,2, \ldots, n\}, \Delta_{2}=\{n+1$, $2, \ldots, 2 \mathrm{n}\}, \Delta_{3}=\{2 \mathrm{n}+1,2 \mathrm{n}+2, \ldots, 3 \mathrm{n}\}$. And let $\mathrm{g}=(1, \mathrm{n}+1$, $2 \mathrm{n}+1)(2, \mathrm{n}+2,2 \mathrm{n}+2)(\mathrm{n}, 2 \mathrm{n}, 3 \mathrm{n})$.

Then $\left(\Delta_{1}\right)^{\mathrm{g}}=\Delta_{2},\left(\Delta_{2}\right)^{\mathrm{g}}=\Delta_{3},\left(\Delta_{3}\right)^{\mathrm{g}}=\Delta_{1}$, so $\mathrm{g}$ interchange $\Delta_{1}$, $\Delta_{2}, \Delta_{3}$, then $\mathrm{g}$ interchange $\operatorname{Sym}\left(\Delta_{1}\right), \operatorname{Sym}\left(\Delta_{2}\right), \operatorname{Sym}\left(\Delta_{3}\right)$. Therefore, $\langle\mathrm{g}\rangle$ acting on the group $\operatorname{Sym}\left(\Delta_{1}\right), \operatorname{Sym}\left(\Delta_{2}\right)$, $\operatorname{Sym}\left(\Delta_{3}\right)$, so you can do the semiproduct of $\langle\mathrm{g}\rangle$ and $\operatorname{Sym}\left(\Delta_{1}\right)$, $\operatorname{Sym}\left(\Delta_{2}\right), \operatorname{Sym}\left(\Delta_{3}\right)$.

Let

$$
\begin{aligned}
G & =\left\langle\operatorname{Sym}\left(\Delta_{1}\right) \times \operatorname{Sym}\left(\Delta_{2}\right) \times \operatorname{Sym}\left(\Delta_{3}\right), g\right\rangle \\
& =\left(\operatorname{Sym}\left(\Delta_{1}\right) \times \operatorname{Sym}\left(\Delta_{2}\right) \times \operatorname{Sym}\left(\Delta_{3}\right)\right):\langle g\rangle \\
& =S_{n} w r C_{3} .
\end{aligned}
$$

Now it is easy to see that $\mathrm{G}$ act on $\Omega$ transitively, but $\mathrm{G}$ is not a primitive group, because it has nontrivial blocks $\Delta_{1}, \Delta_{2}$, $\Delta_{3}$.

Next we consider the following group:

$G_{1}=S_{\{2,3, \cdots, n\}} \times S_{\{n+1, n+2, \cdots, 2 n\}} \times S_{\{2 n+1,2 n+2, \cdots, 3 n\}}=S_{n-1} \times S_{n} \times S_{n}$,

We have 


$$
\begin{gathered}
1^{G_{1}}=\{1\}, \\
2^{G_{1}}=\{2,3, \cdots, n\}, \\
(n+1)^{G_{1}}=\{n+1, n+2, \cdots, 2 n\}, \\
(2 n+1)^{G_{1}}=\{2 n+1, n+2, \cdots, 3 n\} .
\end{gathered}
$$

So the rank of $\mathrm{G}$ is 4 and the subdegrees of $\mathrm{G}$ are $1, n-1, n$, n.

For the group $G=S_{n} w r C_{3}<K=S_{n} w r S_{3}$, it is easy to see that $\mathrm{K}$ also acts on $\Omega$ imprimitively and the orbitals of $\mathrm{K}$ acting on $\Omega$ has the some form as the orbitals of $G$ acting on $\Omega$. So the rank of $\mathrm{K}$ is 4 and the subdegrees of $\mathrm{K}$ are $1, n-1, n, n$, too.

From the above proof, it can be seen that as long as the subgroup of $\mathrm{K}$ satisfies the condition of fixing the set $\left\{\Delta_{1}, \Delta_{2}, \Delta_{3}\right\}$, then the orbitals of its acting on $\Omega$ has the some form as the orbitals of $\mathrm{G}$ acting on $\Omega$. Therefore, the subgroups $\left(\mathrm{S}_{\mathrm{n}}\right.$ wr $\left.\mathrm{S}_{3}\right) \cap \mathrm{A}_{\mathrm{n}}$ and $\left(\mathrm{S}_{\mathrm{n}}\right.$ wr $\left.\mathrm{C}_{3}\right) \cap \mathrm{A}_{\mathrm{n}}$ are all imprimitively act on the $\Omega$ and all of their ranks are 4 and all of their subdegrees are $1, \mathrm{n}-1, \mathrm{n}, \mathrm{n}$.

Corollary 3.4 For $\mathrm{m} \geq 2$, the group $\mathrm{G}=S_{n} w r S_{m}$ and its subgroup $S_{n} w r C_{m}, \quad\left(S_{n} w r S_{m}\right) \cap A_{m},\left(S_{n} w r C_{m}\right) \cap A_{n}$ are imprimitively act on $\Omega=\{1,2, \ldots, \mathrm{mn}\}$. And their rank are all $\mathrm{m}+1$, their subdegree all are $1, \mathrm{n}-1, \underbrace{n, n \cdots, n}_{m-1}$. In addition, when $\mathrm{n}$ is even or $\mathrm{m}$ is odd, the rank of its subgroup $S_{n} w r A_{m}, \quad\left(S_{n} w r \mathrm{~A}_{m}\right) \cap A_{n}$ are all $\mathrm{m}+1$ and their subdegree all are $1, \mathrm{n}-1, \underbrace{n, n, \cdots, n}_{m-1}$.

Proof Let $\Delta_{1}=\{1,2, \ldots, n\}, \Delta_{2}=\{\mathrm{n}+1,2, \ldots, 2 \mathrm{n}\}, \ldots, \Delta_{\mathrm{m}}=$ $\{(\mathrm{m}-1) \mathrm{n}+1,(\mathrm{~m}-1) \mathrm{n}+2, \ldots, \mathrm{mn}\}$. And let $\mathrm{g}=(1, \mathrm{n}+$ $1, \ldots,(m-1) n+1)(2, n+2, \ldots,(m-1) n+2)(n, 2 n, \ldots, m n)$.

$\mathrm{G}:=\left\langle\left(\operatorname{Sym}\left(\Delta_{1}\right) \times \operatorname{Sym}\left(\Delta_{2}\right) \times \ldots \times \operatorname{Sym}\left(\Delta_{\mathrm{m}}\right)\right), \mathrm{g}\right\rangle=\mathrm{S}_{\mathrm{n}} \mathrm{wr}$ $\mathrm{C}_{\mathrm{m}}$.

Obviously $\mathrm{G}$ is imprimitively on $\Omega$ with nontrivial block $\Delta_{1}, \Delta_{2}, \ldots, \Delta_{\mathrm{m}}$.

Then we have,

$$
\begin{gathered}
G_{1}=S_{n-1} \times S_{n} \times S_{n} \\
1^{G_{1}}=\{1\}, \\
2^{G_{1}}=\{2,3, \cdots, n\},
\end{gathered}
$$$$
\left|(1, j, k)^{L}\right|=\left\{\left(1^{g_{k}}, j^{g_{k}}, k^{h} \mid g_{k} \in S_{m}, 1^{h}=1\right\}|=|\{(s, t, v) \mid v \in 1\} \mid=m^{2}(m-1),\right.
$$$$
\left|(i, j, 1)^{L}\right|=\left\{\left(i^{g_{1}}, j^{g_{1}}, 1^{h} \mid 1^{g_{1}}=1^{h}=1\right\}|=\{(s, t, 1) \mid s \in 1, t \in 1\}|=(m-1)(m-1),\right.
$$$$
\left|(\mathrm{i}, 1,1)^{L}\right|=\mid\left\{\left(i^{g_{1}}, 1^{g_{1}}, 1^{h} \mid 1^{g_{1}}=1^{h}=1\right\}|=\{(s, 1,1) \mid s \in 1\}|=(m-1)\right.
$$

Therefore the rank of $\mathrm{G}$ is $\mathrm{m}+1$ and the subdegree of $\mathrm{G}$ is $1, n-1, \underbrace{n, n, \cdots, n}_{m-1}$.

While the group $G=S_{n} w r C_{m} \in K=S_{n} w r S_{m}$, it is easy to show that the group $\mathrm{K}$ imprimitively act on $\Omega$, and the orbit of its point stabilizer acting on $\Omega$ has some form with $G$. Therefore the rank of $\mathrm{K}$ is $\mathrm{m}+1$ and the subdegree of $\mathrm{K}$ is 1 , $\mathrm{n}-1, \underbrace{n, n, \cdots, n}_{m-1}$.

Otherwise, the subgroups $\left(\mathrm{S}_{\mathrm{n}}\right.$ wr $\left.\mathrm{S}_{\mathrm{m}}\right) \cap \mathrm{A}_{\mathrm{n}},\left(\mathrm{S}_{\mathrm{n}}\right.$ wr $\left.\mathrm{C}_{\mathrm{m}}\right) \cap$ $\mathrm{A}_{\mathrm{n}}$, are imprimitively act on $\Omega$ and the orbit of its point stabilizer acting on $\Omega$ has some form with $G$. Therefore the rank of $\mathrm{K}$ is $\mathrm{m}+1$ and the subdegree of $\mathrm{K}$ is $1, \mathrm{n}-1, \underbrace{n, n, \cdots, n}_{m-1}$.

when $\mathrm{n}$ is even or $\mathrm{m}$ is old, let $\mathrm{g}=(1, \mathrm{n}+1, \ldots,(\mathrm{m}-1) \mathrm{n}+$ $1)(2, \mathrm{n}+2, \ldots,(\mathrm{m}-1) \mathrm{n}+2) \ldots(\mathrm{n}, 2 \mathrm{n}, \ldots, \mathrm{mn}) \in \mathrm{A}_{\mathrm{m}}$. $g=(1, n+1, \cdots,(m-1) n+1)(2, n+2, \cdots,(m-1) n+2) \cdots(n, 2 n, \cdots, m n) \in \mathrm{A}_{m}$

Then the subgroup $S_{n}$ wr $A_{m}$ and $\left(S_{n}\right.$ wr $\left.A_{m}\right) \cap A_{n}$ all are imprimitively act on $\Omega$, and the orbit of its point stabilizer acting on $\Omega$ has some form with $\mathrm{G}$. Therefore the rank of $\mathrm{K}$ is $\mathrm{m}+1$ and the subdegree of $\mathrm{K}$ is $1, \mathrm{n}-1, \underbrace{n, n, \cdots, n}_{m-1}$.

Theorem 3.5 The group $S_{m} w r S_{m}$ has an imprimitive action of degree $\mathrm{m}^{3}$ with rank 5 , and the subdegrees are 1 , $\mathrm{m}-1, \mathrm{~m}-1,(\mathrm{~m}-1)(\mathrm{m}-1), \mathrm{m}^{2}(\mathrm{~m}-1)$.

Proof Let $\Delta=\{1,2, \ldots, \mathrm{m}\}$ and $\Omega=\Delta \times \Delta \times \Delta$. The action of group $G=S_{m} w r S_{m}$ in $\Omega$ is as follows:

$$
(\mathrm{i}, j, k)^{\left(g_{1}, g_{2}, \cdots, g_{m}, h\right)}=\left(i^{g_{k}}, j^{g_{k}}, k^{h}\right),
$$

where $\mathrm{i}, j, k \in \Delta ; g_{1}, g_{2}, \cdots, g_{m} \in S_{m} ; h \in S_{m}$.

Let $\alpha=(1,1,1) \in \Omega$, then the stabilizer of $\alpha$ in $\mathrm{G}$ is

$$
G_{\alpha}=L=\left\{\left(g_{1}, g_{2}, \cdots, g_{m}, h\right)||^{g_{1}}=1^{h}=1\right\} .
$$

For the integer $\mathrm{i}>1$, we have 


$$
\left|(1, j, 1)^{L}\right|=\left\{\left(1^{g_{1}}, j^{g_{1}}, 1^{h} \mid 1^{g_{1}}=1^{h}=1\right\}|=|\{(1, t, 1) \mid t \in 1\} \mid=(m-1)\right.
$$

so the rank of $\mathrm{G}$ is 5 and the subdegree is $1, \mathrm{~m}-1, \mathrm{~m}-1$, $(\mathrm{m}-1)(\mathrm{m}-1), \mathrm{m}^{2}(\mathrm{~m}-1)$.

Corollary 3.6 For $\mathrm{n}<\mathrm{m}$, the group $\mathrm{G} S_{m} w r S_{m}$ has an imprimitive action of degree $\mathrm{m}^{\mathrm{n}}$ with rank $2 \mathrm{n}+1$.

Proof Let $\Delta=\{1,2, \cdots, \mathrm{n}\}$, and $\Omega=\underbrace{\Delta \times \Delta \times \cdots \times \Delta}_{\mathrm{n}}$. The action of group $G=S_{m} w r S_{m}$ in $\Omega$ is as follow:

$$
\left(\mathrm{i}_{1}, i_{2}, \cdots, i_{n}\right)^{\left(g_{1}, g_{2}, \cdots, g_{m}, h\right)}=\left(i_{1}^{g_{k}}, i_{2}^{g_{k}}, \cdots, i_{n-1}^{g_{k}}, i_{n}^{h}\right),
$$

where $\mathrm{i}_{1}, i_{2}, \cdots, i_{\mathrm{n}} \in \Delta ; g_{1}, g_{2}, \cdots, g_{m} \in S_{m} ; h \in S_{m}$.

Let $\alpha=\underbrace{(1,1, \cdots, 1)}_{n} \in \Omega$, then the stabilizer of $\alpha$ in $\mathrm{G}$ is

$$
G_{\alpha}=L=\left\{\left(g_{1}, g_{2}, \cdots, g_{m}, h\right) \mid 1^{g_{1}}=1^{h}=1\right\} .
$$

For the integer $i_{j}>1, j=1,2, \ldots, n$, we have,

$$
\begin{aligned}
& \left|\left(1, \mathrm{i}_{2}, \cdots, \mathrm{i}_{\mathrm{n}}\right)^{\mathrm{L}}\right| \\
= & \left|\left\{1^{\mathrm{x}},\left(\mathrm{i}_{2}\right)^{\mathrm{x}}, \cdots,\left(\mathrm{i}_{\mathrm{n}-1}\right)^{\mathrm{x}} \mid \mathrm{x}=\mathrm{g}_{\mathrm{n}} \in \mathrm{S}_{\mathrm{m}}, 1^{\mathrm{h}}=1\right\}\right| \\
= & \left|\left\{\left(\mathrm{j}_{1}, \mathrm{j}_{2}, \mathrm{j}_{\mathrm{n}}\right) \mid \mathrm{j}_{\mathrm{n}} \neq 1\right\}\right| \\
= & \mathrm{m}^{(\mathrm{n}-1)}(\mathrm{m}-1) .
\end{aligned}
$$

Now consider the case of $\mathrm{j}_{\mathrm{n}}=1$, according to the proof process of theorem 3.5, if the number and position of non-1 in $i_{1}, i_{2}, \ldots, i_{n-1}$ are different, they will belongs to different suborbit in $\mathrm{G}$.

When you have 0 elements equal to 1 in $i_{1}, i_{2}, \ldots, i_{n-1}$, there are $C_{n-1}^{0}$ suborbits of length $\underbrace{(m-1)(m-1) \cdots(m-1)}_{n-1}$; when you

have 1 elements equal to 1 in $\mathrm{i}_{1}, \mathrm{i}_{2}, \ldots, \mathrm{i}_{\mathrm{n}-1}$, there are $C_{n-1}^{1}$ suborbits of length $\underbrace{(m-1)(m-1) \cdots(m-1)}_{n-2}, \ldots$, when you have n-2 elements equal to 1 in $\mathrm{i}_{1}, \mathrm{i}_{2}, \ldots, \mathrm{i}_{\mathrm{n}-1}$, there are $C_{\mathrm{n}-1}^{n-2}$ suborbits of length $(m-1)$, so the rank of $\mathrm{G}$ is $2^{\mathrm{n}}+1$.

\section{Conclusion}

According to the proof in the third part, we have a imprimitive groups $S_{n}$ wr $S_{3}, S_{n}$ wr $C_{3}$, $\left(S_{n} w r S_{3}\right) \cap A_{n}$, and $\left(\mathrm{S}_{\mathrm{n}}\right.$ wr $\left.\mathrm{C}_{3}\right) \cap \mathrm{A}_{\mathrm{n}}$ with rank 4 acting on $3 \mathrm{n}$ points and a imprimitive group $S_{m}$ wr $S_{m}$ with rank 4 acting on $\mathrm{m}^{3}$ points, where $m$ and $n$ are positive integer.

\section{Funding}

The project was funded by the National Fund NSFC (11561078).

\section{References}

[1] N. Biggs. Algebraic Graph Theory, second ed. Cambridge Unir. Press, New York, 1992.

[2] J. D. Dixon and Mortimer, B. Permutation groups. Graduate Texts in Mathematics, Springer-Verlag (1996), Berlin.

[3] Joanna B. Fawcett, Michael Giudici, Cai Heng Li, Cheryl E. Praeger, Gordon Royle and Gabriel Verret. Primitive permutation groups with a suborbit of length 5 and vertex-primitive graphs of valency 5, Journal of Combinatorial Theory, Series A 157 (2018), 247-266.

[4] D. G. Higman. Finite permutation groups of rank 3. Math. Z. 86 (1964), 145-156.

[5] W. Knapp. On the point stabilizer in a primitive permutation group. Math. Z. 133 (1973), 137-168.

[6] C. H. Li, Z. P. Lu and Dragan Marušič. On primitive permutation groups with small suborbits and their orbital graphs. Journal of Algebra, 279 (2004), 749-770.

[7] C. H. Li, H. S. Sim. On half-transitive metacirculants of prime-power order. J. Combin. Theory Ser. B 81 (2001) 45-51.

[8] P. M. Neumann. Finite permutation groups. edge-coloured graphs and matrices, in: Topics in Group Theory and Computation, Proc. Summer school, University College, Galway, (1977), pp. 82-118.

[9] C. E. Praeger. Primitive permutation groups with a doubly transitive subconstituent. J. Austral. Math. Soc. (scries A) 45 (1988), 66-77.

[10] W. L. Quirin. Primitive permutation groups with small orbitals Math. Z. 122 (1971), 267-274.

[11] C. C. Sims. Graphs and finite permutation groups. Math. Z. 95 (1967), 76-86.

[12] H. Wielant. Finite Permutation Groups. Academic Press, New York, 1964.

[13] J. Wang. The primitive permutation groups with an orbital of length 4. Comm. Algebra 20 (1992), 889-921.

[14] J. Wang. Primitive permutation groups with a solvable subconstituent of degree 5. Beijing Daxue Xuebao Ziran Kexue Ban 31 (1995), 520-526.

[15] J. Wang. Primitive permutation groups with an unfaithful subconstituent containing $\mathrm{A}_{5}$. Algebra Colloq. 3 (1996), 11-18.

[16] W. J. Wong. Determination of a class of primitive permutation groups. Math. Z. 99 (1967), 235-246. 